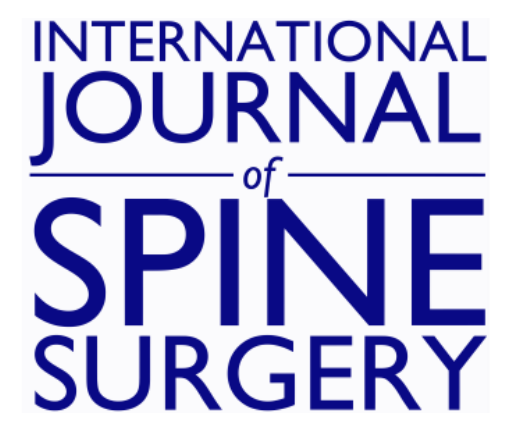

\title{
A Comparison of Open and Percutaneous Techniques in the Operative Fixation of Spinal Fractures Associated with Ankylosing Spinal Disorders
}

Cara L. Sedney, Scott D. Daffner, Abimbola Obafemi-Afolabi, Daniel Gelb, Steven Ludwig, Sanford E. Emery and John C. France

Int J Spine Surg 2016, 10 ()

doi: https://doi.org/10.14444/3023

http://ijssurgery.com/content/10/23

This information is current as of April 25, 2023.

Email Alerts Receive free email-alerts when new articles cite this article. Sign up at:

http://ijssurgery.com/alerts

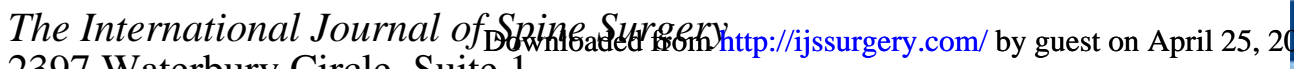
2397 Waterbury Circle, Suite 1,

Aurora, IL 60504, Phone: +1-630-375-1432

(C) 2016 ISASS. All Rights Reserved. 


\section{A Comparison of Open and Percutaneous Techniques in the Operative Fixation of Spinal Fractures Associated with Ankylosing Spinal Disorders}

Cara L. Sedney, MD, MA, ${ }^{1}$ Scott D. Daffner, MD, ${ }^{2}$ Abimbola Obafemi-Afolabi, MD, ${ }^{3}$ Daniel Gelb, MD, ${ }^{3}$ Steven Ludwig, MD, ${ }^{3}$ Sanford E. Emery, MD, $M B A,{ }^{2} J o h n$ C. France, $M D^{2}$

1 Department of Neurosurgery; West Virginia University, Morgantown, WV, 2 Department of Orthopaedics, West Virginia University, Morgantown, WV, ${ }^{3}$ Department of Orthopaedics; University of Maryland, Baltimore, $M D$

\section{Abstract}

Background

The operative care of patients with ankylosing spinal conditions such as ankylosing spondylitis (AS) and diffuse idiopathic skeletal hyperostosis (DISH) after a spine fracture is not well represented in the literature. This work seeks to determine the effect of minimally invasive techniques on patients with spinal fractures and ankylosing spinal conditions through a retrospective case-control analysis.

Methods

The operative logs from 1996-2013 of seven fellowship-trained spine surgeons from two academic, Level I trauma centers were reviewed for cases of operatively treated thoracic and lumbar spinal fractures in patients with ankylosing spinal disorders.

Results

A total of 38 patients with an ankylosing spinal condition and a spinal fracture were identified. The minimally invasive group demonstrated a statistically significant decrease in estimated blood loss, operative time, and need for transfusion when compared to either the hybrid or open group. There was no difference between the three subgroups in overall hospital stay or mortality.

\section{Conclusions}

Patients with ankylosing spinal conditions present unique challenges for operative fixation of spinal fractures. Minimally invasive techniques for internal fixation offer less blood loss, operative time, and need for transfusion compared to traditional techniques; however, no difference in hospital stay or mortality was reflected in this series of patients. Level of Evidence: 4.

Clinical Relevance

Ankylosing spinal disorders are increasingly common in an aging population.

KEYWORDS: SPINAL FUSION, ANKYLOSING SPONDYLITIS, MINIMALLY INVASIVE

VOLUME 10 ARTICLE 23 DOI: 10.14444/3023

\section{Introduction}

The operative care of patients with ankylosing spinal conditions such as ankylosing spondylitis (AS) and diffuse idiopathic skeletal hyperostosis (DISH) after a spine fracture is not well represented in the literature. It is well known that, due to autofusion of the spine in these disease processes resulting in long lever arms and abnormal rigidity, severe fractures with neurologic compromise may occur from even minor trauma. ${ }^{1}$ These patients also tend to be of advanced age with multiple comorbidities, including an increased predisposition to intra-operative bleeding. Minimally invasive surgery (MIS) of the spine is a theoretically attractive option in these patients because of the documented shorter operative times, decreased blood loss, and other factors. These techniques have been described in populations of patients with ankylosing spinal conditions; however, to our knowledge, no one has directly compared open tech- 
niques to minimally invasive techniques. The purpose of this study was to compare two such techniques.

\section{Materials and Methods}

After approval by the Institutional Review Board, the operative logs from 1996 to 2013 of seven fellowshiptrained spine surgeons from two academic, Level I trauma centers were reviewed for cases of operatively treated thoracic and lumbar spinal fractures in patients with AS and DISH. The patients were treated according to the preferences of the attending physician and were stratified according to technique of fixation. The medical records of these patients were reviewed for age, gender, injury mechanism, injury severity score (ISS), fracture type and level, surgical procedure, surgical levels, estimated blood loss, need for transfusion, duration of surgery, complications, days of ventilation, presence of hospital acquired pneumonia, length of hospital stay, maintenance of correction, bony fusion, and mortality.

\section{Statistical Methods}

Statistical analysis was carried out using JMP Version 11 software (SAS, Cary, NC). Analysis of variance (ANOVA) was carried out for continuous variables while contingency tables were used for discrete variables.

\section{Results}

A total of 38 patients with an ankylosing spinal condition and a spinal fracture were identified. One patient with a $\mathrm{T} 1$ fracture was excluded from analysis because of the need to extend fixation into the cervical spine. Of the remaining 37 patients, 14 underwent an open fusion surgery, 19 underwent a minimally invasive internal fixation, and four underwent a hybrid procedure. Hybrid procedures included a minimally invasive instrumentation component with an open component due to either system malfunction or desire for a formal fusion procedure at a localized spinal level, similar to the technique previously described in a case report by Sebastian and colleagues. ${ }^{2}$

These three groups were analyzed both as a whole and as separate subgroups. Average age across groups was 74 (range 41-90 years) with no significant difference between groups. Sixty-five percent were male and the ISS was similar across groups. The most common mechanism was a fall; low-energy mechanisms and missed injuries were common, with delayed diagnosis in seven cases. In three cases, no specific traumatic event was recalled by the patients, who were often admitted for medical problems, but imaging demonstrated an acute fracture. Extensiontype injuries were the most common fracture type. Four patients sustained fractures in two separate locations. The majority were thoracic level fractures (33 patients). Five patients required delay of surgery due to active medical issues such as cardiac events or hemodynamic instability from urosepsis or liver failure at the time of admission. The average number of levels operated on was 5.2.

Five patients died either in the initial hospitalization or during follow-up (three during hospitalization and two patients within three months post-operatively, all from medical complications). Inpatient mortalities were due to failure of extubation in a "do not reintubate" patient, multiorgan system failure related to trauma, and fulminant liver failure due to preexisting cirrhosis. Follow-up was available in $24 \mathrm{pa}-$ tients. Average follow-up was 18.2 months for those patients (range 6 weeks to 60 months). Follow-up imaging was available in 14 patients and demonstrated adequate fracture healing without instrumentation malfunction in any patient. Two patients treated with open fusion and one hybrid patient required wound washout due to infection. One patient (in the open group) had instrumentation removal due to prominence after fracture healing. One patient had a subsequent fall and fracture eight months from the index procedure which required additional operative fixation.

When the three subgroups were compared, the minimally invasive group demonstrated a statistically significant decrease in estimated blood loss (Figure 1; $\mathrm{p}<0.0001$ ) as well as operative time (Figure 2; $\mathrm{p}=0.0003$ ) and need for transfusion (Figure 3; $\mathrm{p}=0.0014$ ) when compared to either the hybrid or open group. The hybrid group was consistently similar to the open group in this regard (Figure 1, Figure 2 , Figure 3). There was no difference between the 
three subgroups in overall hospital stay or mortality. These findings were preserved when controlled for ISS.

\section{Discussion}

Benefits of MIS

Minimally invasive spinal surgery is becoming increasingly popular, with the theoretical advantages that it diminishes estimated blood loss and operative
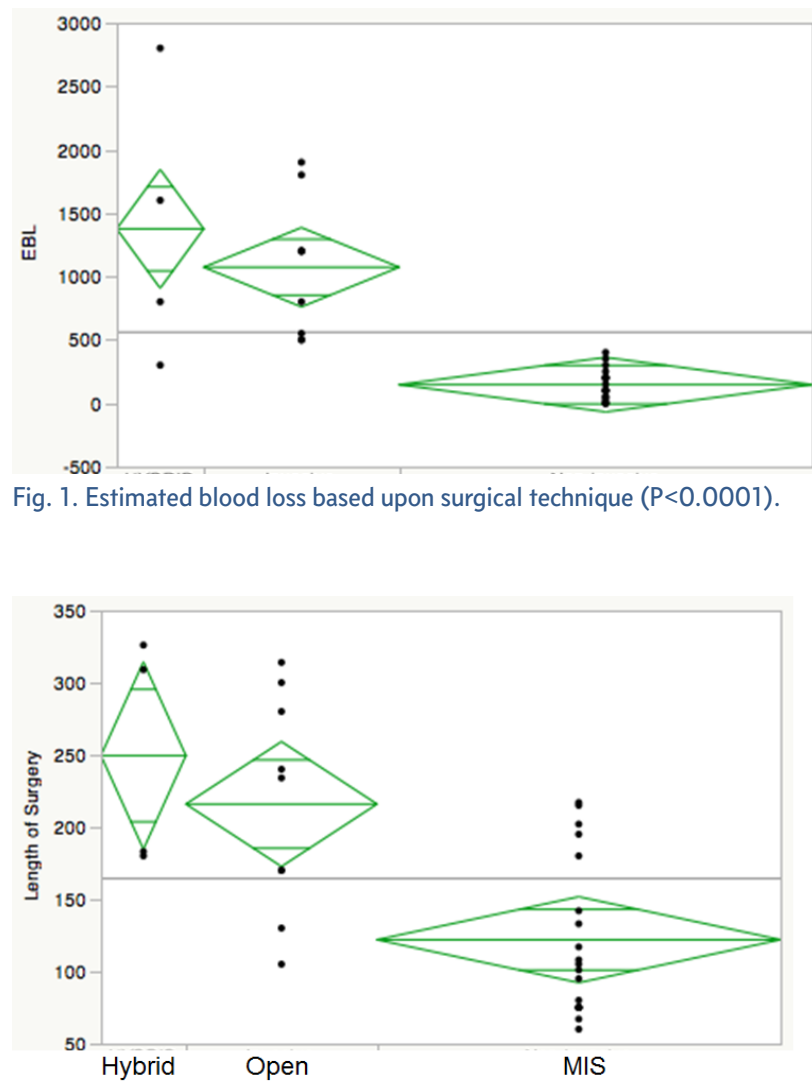

Fig. 2. Operation time based upon surgical technique $(P=0.0003)$.

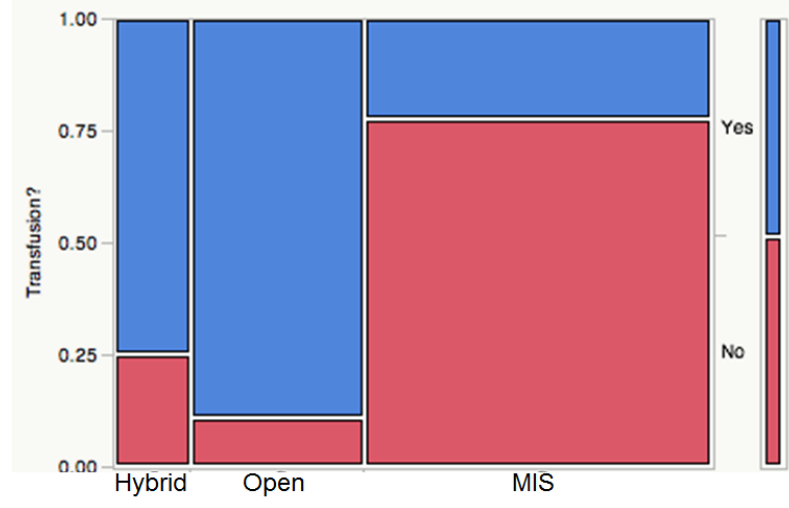

Fig. 3. Need for transfusion based upon surgical technique $(P=0.0014)$. time as well as immediate post-operative pain scores. However, the utility and exact indications for these techniques have not been completely elucidated. A number of case series have been published demonstrating acceptable outcomes using minimally invasive techniques for patients with ankylosing spinal disorders; however, a direct comparison with open techniques has not been performed. Our findings demonstrate that patients with AS and DISH with a spinal fracture had decreased blood loss, decreased need for transfusion, and decreased operative time using MIS techniques compared to open treatments. However, we were not able to demonstrate a difference in overall length of hospital stay or mortality. These findings are comparable to the findings of other groups exploring the utility of MIS techniques for various indications.

The characteristics of patients with ankylosing spinal disorders, such as autofusion creating fractures with long lever arms, require techniques more similar to long bone fracture fixation. Specifically, multiple points of fixation are required for stability. Furthermore, the tendency of ankylosing patients to autofuse lends a theoretical advantage to MIS techniques with osteosynthesis (rather than arthrodesis), which are essentially internal fixation techniques. In both previous literature and in our case series, these patients are elderly with multiple medical comorbidities, ${ }^{3}$ making the proposed benefits of MIS techniques valuable.

\section{Previous Literature for AS}

Recently, two case series, both consisting of ten patients, have been published regarding the use of MIS techniques in the operative fixation of spinal fractures in patients with ankylosing spinal conditions. Yeoh and colleagues reported on a retrospective series of ten patients with ankylosing spinal disorders, treated with MIS techniques after a spinal fracture. Similar to our series, delayed diagnosis was common. They reported acceptable pain outcomes using the Oswestry disability index and visual analog scale out to 22 months, with no patient lost to follow-up, although two patients died. ${ }^{4}$ Kruger and colleagues similarly reported on a series of ten patients with ankylosing spinal disorders treated with MIS instrumentation after a thoracolumbar fracture. They 
found acceptable outcomes as defined by ambulation ability and pain upon follow-up. ${ }^{5}$ Prior to these case series, only single case reports have been present in the literature. Our series is the largest to date and the only study to directly compare open and minimally invasive techniques in this patient population.

\section{Epidural Hematoma}

The increased risk of epidural hematoma in patients with ankylosing spondylitis is well-reported. ${ }^{1}$ Two patients in our series required an open laminectomy for decompression of an epidural hematoma. In these patients (both in the MIS group), the addition of an open laminectomy procedure did not add significantly to the operative time or blood loss.

\section{Hybrid Techniques}

The use of hybrid techniques as described by Sebastian, ${ }^{2}$ for instance an open fusion at specific levels (a patient with two operative fractures, one of which was amenable to internal fixation and one which required fusion) with minimally invasive instrumentation placement, might be postulated to accomplish the operative goals of a formal fusion procedure while preserving the benefits of minimally invasive techniques. In our case series, one patient planned as MIS required an open procedure due to system malfunction, while three underwent a planned hybrid procedure due to multiple fractures, one requiring fusion and one amenable to internal fixation only. We found that hybrid procedures had a similar profile as compared to open procedures.

\section{Delayed Diagnosis}

Because of the many low-energy injuries and subtle radiologic findings, spinal fractures are often missed in this population of patients. ${ }^{6}$ In our patient population, low-energy mechanisms were common, such as falls from standing. In several cases, no specific traumatic event was recalled by the patient. A delayed diagnosis was seen frequently in our patient population (14\% of patients), as previously reported by Scheifer and colleagues. ${ }^{6}$

\section{Limitations}

Our study, although it reports on the largest number of patients treated for spine fractures with ankylosing spinal conditions to date, suffers from the limitations of its retrospective nature as well as its poor followup, making meaningful statistical analysis or conclusions regarding the post-hospitalization course and ultimate efficacy difficult. Additionally, specific outcome measures would have improved the quality of these data and given some indication of long-term benefits. Nevertheless, we feel the use of MIS techniques appears to be a viable option in this difficult patient population.

\section{Conclusions}

Patients with ankylosing spinal conditions present unique challenges for operative fixation of spinal fractures. Minimally invasive techniques for internal fixation offer less blood loss, operative time, and need for transfusion compared to traditional techniques; however, no difference in hospital stay or mortality was reflected in this series of patients. Overall, MIS techniques may be a viable option for consideration in this difficult patient population.

\section{Acknowledgement}

The authors wish to acknowledge Gerald Hobbs, $\mathrm{PhD}$, Nina Clovis, Suzanne Danley, and Sheila Rye, MS, for their assistance with the preparation of this paper.

\section{References}

1. Choi D. Fractures in ankylosing disorders of the spine; easy to miss and high risk of deterioration. World Neurosurg. 2015;83:6.1029-31. doi: 10.1016/j.wneu.2015.01.046.

2. Sebastian AS, Fogelson JL, Dekutoski MB. Et al. Multiple noncontiguous spinal fractures and occipitocervical dislocation in a patient with ankylosing spondylitis treated with a hybrid open and percutaneous spinal fixation technique: a case report. Spine J. 2015;15:5e.e1-5. doi: 10.1016/

j.spinee.2015.02.005.

3. Lee JK, Park KS, Park MS, et al. Surgical treatment of lumbar hyperextension injury in ankylosing spondylitis. Korean J Spine. 2013;10:3.195-9. doi: 10.14245/kjs.2013.10.3.195

4. Yeoh D, Moffatt T, Karmani S. Good outcomes 
of percutaneous fixation of spinal fractures in ankylosing spinal disorders. Injury. 2014;45:10.1534-8. doi: 10.1016/j.injury.2014.03.020.

5. Kruger A, Frink M, Oberkircher L, et al. Percutaneous dorsal instrumentation for thoracolumbar extension-distraction fractures in patients with ankylosing spinal disorders: a case series. Spine J. 2014;14:12.2897-904. doi: 10.1016/ j.spinee.2014.04.018. 6. Schiefer TK, Millian BD, Bracken CD, et al. Inhospital neurologic deterioration following fractures of the ankylosed spine: a single-institution experience. World Neurosurg. 2015;83:5.775-83. doi: 10.1016/j.wneu.2014.12.041

\section{Disclosures \& COI}

Daffner reports research support from Pfizer, research support from Bioventus, research support from Spinal Kinetics, grants from AO North Ameri$\mathrm{ca}$, grants from CSRS, grants from AO Spine, stock in Pfizer, stock in Amgen, and receiving personal fees from DePuy Synthes Spine. Emery reports no conflicts of interest. France reports no conflicts of interest. Gelb reports no conflicts of interest. Ludwig is an AAOS board member; AOA board member; has recived research support from the AOSNA spine fel- lowship; owns stock in ASIP \& ISD; is a board or committee member for CSRS; receives royalties, consults for, and is a paid presenter for DePuy Synthes Spine; has received research support from and been paid consultant to Globus Medical; is on the JSDT Editorial Board; research support and paid consultant to K2M Spine; research support from OMEGA; research support from Pacira; is a board or committee member ofr SMISS, is a paid consultant/ speaker for Synthes Spine, receives royalties from Thieme/QMP publishing. Obafemi reports no conflicts of interest. Sedney reports no conflicts of interest.

\section{Corresponding Author}

Cara L. Sedney, MD, MA, Department of Neurosurgery; West Virginia University, PO Box 9183, Morgantown, WV 26506.

Published 7 June 2016.

This manuscript is generously published free of charge by ISASS, the International Society for the Advancement of Spine Surgery. Copyright ๑ 2016 ISASS. To see more or order reprints or permissions, see http://ijssurgery.com. 